\title{
Effective AL-FEC with LDGM for Interactive Video Over DSL
}

\author{
Laith A1-Jobouri , Filippo Casu , Martin Fleury , and Julián Cabrera
}

\begin{abstract}
Interactive video, due to the need to maintain synchronization, has strict latency requirements. Video streaming to residential users is also constrained by the error bursts that are a feature of Digital Subscriber Link (DSL) access. This paper examines the video response to unlicensed LowDensity Generator Matrix (LDGM) channel codes that combine reduced computational latency and small block sizes with reasonable recovery properties. The paper demonstrates that up to $4 \mathrm{~dB}$ gain in video quality is achievable with LDGM Forward Error Correction (FEC) at the Application Layer (AL).
\end{abstract}

\section{INTRODUCTION}

Digital Subscriber Line (DSL) is the dominant broadband access network for residential users with 364.1 million links in 2012 [1]. However, impulse noise is a potent source of DSL transmission errors, resulting in fixed-length error bursts consisting of multiple packet losses. Consequently, an active area of research [2] [3] is how best to protect interactive video services such as home-based video conferencing. The preferred solution is Application-Layer (AL)-Forward Error Correction (FEC), because such video streaming applications cannot tolerate repeat transmissions at the data-link layer. ALFEC tends to operate at the packet level, assuming that physical-layer FEC will correct bit errors within a packet or fail to do so, resulting in a packet erasure.

Channel coding (FEC) latency should be minimized to allow the synchronization of interactive video streams. Coding latency is composed of: the delay owing to: the need to accumulate sufficient data to successfully repair packets, a problem that may arise with the rateless codes in [2], leading to large input buffers; and the delay arising from the computational complexity of the coding and decoding operations, a problem with Reed-Solomon (RS) code [4] as the block size increases.

To this end, Low-Density Generator Matrix (LDGM) codes [5], a low-complexity version of Low-Density Parity Check (LDPC) codes, are attractive candidates. They are block-based but unlike LDPC codes, which can have block sizes of about 1000 for best recovery performance [5], LDGM can use lower values of $k$ (see next paragraph), reducing latency. Their reduced coding complexity can be traded against their errorcorrection performance. Unlike RS codes, decoding can be iteratively refined through a belief-propagation algorithm, rather than wait for all the data before decoding can begin. As in both [3] and [6], LPDC was selected as most suitable for Real-time Transport Protocol (RTP) video streaming, the possibilities for its simplified cousin, LDGM, are promising.
While our previous work compared LDGM to other FEC options [7], in this paper the video response is demonstrated, directly highlighting LDGM's potential. Prior work by others [3] [6] did not examine the video quality response.

LPDC and LDGM belong to the class of linear block codes that are defined by parameters: $k$, the number of nodes of a data vector; and by $n$, the number of nodes of a code vector. In our case of a packet erasure channel, each node is a packet. The number of redundant nodes (parity nodes) is $\mathrm{n}-\mathrm{k}$ that, in the case of a systematic code, is concatenated with the $\mathrm{k}$ nodes of a data vector. An LPDC code is defined by its parity-check matrix $H$ of dimensions $n-k$ by $n$, of which entries are exclusively 1 's and 0 's and obey equation (1):

$$
\mathrm{cHT}=0
$$

where $\mathrm{c}$ is the code vector. The sparse entries of $\mathrm{H}$ are randomly specified. We used a regular, staircase matrix layout [3] [6], in which the number of 1's in each row and column is constant. $H$ provides $n-k$ parity check equations that generate constraints between data nodes and parity nodes, which indicate which data nodes are involved in the XOR operations that generate the parity nodes. From $\mathrm{H}$ we are able to construct the generator matrix $G$ that allows the creation of the parity nodes from the data vector following equation (2):

$$
\mathbf{c}=\mathbf{u G}
$$

where $\mathrm{c}$ is the code vector ( $\mathrm{n}$ bits), and $\mathrm{u}$ the data vector $(\mathrm{k}$ bits). Thus, matrix $\mathrm{G}$ has a size of $\mathbf{k}$ by $\mathbf{n}$. Though LPDC and LDGM are not maximum distance separable codes, implying more than $\mathrm{k}$ symbols need to be received to allow reconstruction, only around $\mathrm{k} \times 1.05 \%$ symbols need in practice to be received [6], making the case for lower block sizes.

In LDGM, $G$ is generated directly from $H$, involving a lower number of operations with respect to the LDPC case. For this reason, LDGM codes lead to lower encoding and decoding computational times. In the LDGM approach, the parity-check matrix $H$ has a size of $n-k$ by $k$, compared to the LPDC case above of $n-k$ by $n$. Therefore, only the first $k$ nodes and their coding contribution are taken into consideration, compared to the LDPC case, for which n nodes have to be handled. LDPC has a superior recovery performance because parity packets are better protected but the reduced number of coding operations compensates for this short-coming when employing LDGM. LDGM shares with LDPC XOR operations guided by sparse, random, binaryvalued matrices. In [7] it was shown that for error burst lengths of 20 and 25 packets, LDGM's error-correction 
performance was better than the well-known XOR interleaving matrix method COP \#3. The poorer overall performance of such 2D parity codes is confirmed in [3] and [8]. In this short paper, we show that, for a DSL channel, the objective video quality reaches broadcast quality when using LDGM.

\section{METHODOLOGY}

In experiments, Asymmetric DSL (ADSL) was assumed with small packet sizes of $50 \mathrm{~B}$ and $100 \mathrm{~B}$ for the two sets of tests respectively. The former size is close to the $53 \mathrm{~B}$ cell size of the Asynchronous Transfer Mode (ATM) predominantly employed over ADSL. (Only 48 bytes (B) form the payload in ATM; the remaining header bytes are heavily protected.) ADSL "Fast Track" was turned on but packet interleaving to reduce burst lengths was turned off so as to reduce latency. For $100 \mathrm{~B}$ packets a download bitrate of $10 \mathrm{Mbps}$ was set, with a per packet link latency of around $100 \mathrm{~ms}$. For $50 \mathrm{~B}$ packets and two-way communication 1 Mbps effective datarate was assumed, with a per packet link latency of around $10 \mathrm{~ms}$. The packet error rate was set to a typical ADSL worst case of $1 \%$ (see [8]) with burst lengths of 8 and 10 packets for the $50 \mathrm{~B}$ and $100 \mathrm{~B}$ packets respectively. Bursts occurred randomly (Uniform distribution).

To counter the error bursts, packets were grouped into $k$ packets at a time with the addition of $9 \%$ redundant packets. $k$ $=300,400$, well below $k=1000$ in large block size coding schemes [5]. The reference video clip Football, with plenty of motion activity, which increases the inter-packet coding dependencies, was encoded with the H.264/Advanced Video Coding (AVC) JM 14.2 codec in Common Intermediate Format $(352 \times 288$ pixels/frame $)$ at 30 frames $/ \mathrm{s}$ at a constant bitrate of $1 \mathrm{Mbps}$. The frame structure was an initial intracoded frame followed by all predictively coded P-frames. $2 \%$ intra-coded macroblocks were added to the P-frames to check temporal error propagation. Data-partitioning was turned on at the codec as an additional form of error resilience, with constrained intra prediction also configured. These video settings conform to the recommendations of [9].

\section{RESULTS}

In order to judge the video quality, a video trace was fed into a numerical simulator where ADSL packetization took place. After numerical simulation, data from the ADSL packets judged lost were removed from the compressed video bitstream, prior to passing through the H.264/AVC decoder. The resulting bitstreams (before and after LDGM repair) were compared to the YUV video input to determine the PSNR.

Table I shows 5 sample runs each with a different seed and the resulting mean PSNR. (The code seed was set to 40, 50, 70,80 , and 90 for $1-5$ respectively.) The mean gain after application of LDGM is between 1 and $2 \mathrm{~dB}$ so that the video quality approaches broadcast quality. Interestingly from the point of view of latency, increasing the block size does not necessarily lead to an improvement in video quality. For the larger packet sizes and the greater bandwidth of Table II, the video quality gain is more consistent and is $3-4 \mathrm{~dB}$. The consistency is due to a constant code seed of 50 throughout. Notice that in view of the larger $100 \mathrm{~B}$ packet size the block sizes are decreased. Again a larger block size appears not to lead to an advantage. This effect may be linked to the pattern of packet burst erasures. Comparing the $100 \mathrm{~B}$ PSNR gain to that of $50 \mathrm{~B}$ packets, for the latter the FEC gain appears to have saturated, suggesting a reduced FEC rate is possible.

TABLE I

OBJECTIVE VIDEO QUALITY FOR 50 B PACKETS BEFORE AND AFTER FEC

\begin{tabular}{|l|c|c|c|c|c|c|}
\hline $\begin{array}{l}\text { Before } \\
\text { FEC }\end{array}$ & 1 & 2 & 3 & 4 & 5 & $\begin{array}{c}\text { Mean PSNR } \\
(\mathrm{dB})\end{array}$ \\
\hline $\mathrm{k}=300$ & 35.53 & 35.08 & 38.63 & 36.14 & 37.37 & 36.55 \\
\hline $\mathrm{k}=400$ & 34.46 & 37.66 & 38.54 & 36.40 & 39.00 & 37.21 \\
\hline After & 1 & 2 & 3 & 4 & 5 & \\
\hline $\mathrm{k}=300$ & 37.33 & 39.00 & 39.00 & 37.57 & 37.84 & 38.15 \\
\hline $\mathrm{k}=400$ & 36.91 & 37.76 & 39.00 & 37.64 & 39.00 & 38.06 \\
\hline
\end{tabular}

TABLE II

OBJECTIVE VIDEO QUALITY FOR 100 B PACKETS BEFORE AND AFTER FEC

\begin{tabular}{|l|c|c|c|c|c|c|}
\hline $\begin{array}{l}\text { Before } \\
\text { FEC }\end{array}$ & 1 & 2 & 3 & 4 & 5 & $\begin{array}{c}\text { Mean PSNR } \\
(\mathrm{dB})\end{array}$ \\
\hline $\mathrm{k}=200$ & 34.53 & 33.95 & 34.91 & 34.93 & 35.64 & 34.79 \\
\hline $\mathrm{k}=300$ & 36.54 & 36.41 & 33.81 & 34.69 & 32.84 & 34.86 \\
\hline After & 1 & 2 & 3 & 4 & 5 & \\
\hline $\mathrm{k}=200$ & 38.22 & 36.67 & 39.00 & 39.00 & 38.86 & 38.35 \\
\hline $\mathrm{k}=300$ & 38.85 & 36.55 & 39.00 & 38.88 & 37.35 & 38.13 \\
\hline
\end{tabular}

\section{CONCLUSION}

Recent studies [6] [7] [2] all show that industry-standard 2D parity codes underperform in terms of combined latency and error recovery. Alternatively, LDGM codes with small block sizes for small DSL packet sizes represent a natural candidate for low-latency interactive video streaming and, as results quoted in this paper indicate, can lead to up to $4 \mathrm{~dB}$ gain in video quality for active sports sequences with a $9 \%$ increase in datarate. Given that rateless codes have patents applied, LDGM codes offer a further commercial advantage.

\section{REFERENCES}

[1] Point Topic Ltd., "World Broadband Statistics: Q2, 2012" London, U.K., 2012.

[2] M. Ellis, D.P. Pezaros, and C. Perkins, "Performance analysis of ALFEC for RTP-based streaming video traffic to residential users," in IEEE $19^{\text {th }}$ Int'l. Packet Video Workshop, 2012.

[3] V. Pullano, R. Skupin, G.E. Corazza, C. Hellge, and T. Schierl, "Modeling effects of impulse noise on application-layer FEC in DSL channels," in IEEE Int'l Symp. Broadband Mult. Syst. Broadcast., 2013.

[4] T. Jokela, and E. Lehtonen, "Reed-Solomon decoding algorithms and their complexities at the DVB-H link-layer," in $4^{\text {th }}$ IEEE Int'l. Symp. Wireless Commun. Systems, pp. 752-756, 2007.

[5] V. Roca, Z. Khalluf, and J. Labouré, "Design and evaluation of an LDGM large block FEC codec," in Fifth Int'l. Workshop on Networked Group Commun., 2003.

[6] K. Matsuzono, J. Detchart, M. Cunche, V. Roca et al. "Performance analysis of a high-performance real-time application with several ALFEC schemes," in IEEE Local Computer Networks, 2010.

[7] F. Casu, J. Cabrera, F. Jaurequizar, and N. Garcia, "Low latency LDGM code for multimedia-packet stream in bursty packet loss networks," in IEEE Int'l. Conf. Consumer Electronics, pp. 63-64, 2012.

[8] M. Luby, T. Stockhammer, and M. Watson, "Application layer FEC in IPTV services," IEEE Commun. Mag., vol. 46, no. 5, pp. 94-101, 2008.

[9] L. Al-Jobouri, M. Fleury, and M. Ghanbari, "Engineering wireless broadband access to IPTV," $J$. of Visual Commun. and Image Representation, vol. 25, no. 7, pp. 1493-1506, 2014. 